\title{
Racial Disparities
}

\section{The Causes of Racial and Ethnic Differences in Influenza Vaccination Rates among Elderly Medicare Beneficiaries}

\author{
Paul L. Hebert, Kevin D. Frick, Robert L. Kane, and A. Marshall \\ McBean
}

Objective. To explore three potential causes of racial/ethnic differences in influenza vaccination rates in the elderly: (1) resistant attitudes and beliefs regarding vaccination by African-American and Hispanic Medicare beneficiaries, (2) poor access to care during influenza vaccination weeks, and (3) discriminatory behavior by providers.

Data Sources. Medicare beneficiaries who responded to both the 1995 and 1996 Medicare Current Beneficiary Survey (MCBS) $(n=6,746)$.

Study Design. We combined survey information from the MCBS with Medicare claims. We measured resistance to vaccination by self-reported reasons for not receiving vaccination, access to care by claims submitted during vaccination weeks, and discrimination by racial differences in vaccinations among beneficiaries who visited the same providers during vaccination weeks.

Principal Findings. White beneficiaries (66.6 percent) were more likely to self-report having received vaccination than were African Americans (43.3 percent) or Hispanics (52.5 percent). Resistance to vaccination plays a role in low vaccination rates of AfricanAmerican ( -11.8 percentage points), but not Hispanic beneficiaries. Unequal access accounts for $<2$ percent of the disparity. Minority beneficiaries remained unvaccinated despite having medical encounters with their usual providers on days when those same providers were administering vaccinations to white beneficiaries. This disparity is attributable not to provider discrimination but to a $1.6-5 \times$ higher likelihood of white beneficiaries initiating encounters for the purpose of receiving vaccination.

Conclusion. Disparities in access to care and provider discrimination play little role in explaining racial/ethnic disparities in influenza vaccination. Eliminating missed opportunities for vaccination in 1995 would have raised vaccination rates in three racial/ethnic groups to the Healthy People 2000 goal of 60 percent vaccination.

Key Words. Influenza, vaccination, racial disparities, preventable diseases, discrimination

Influenza outbreaks occur nearly every year and cause significant excess medical costs (McBean, Babish, and Warren 1993), as well as an average of 36,000 excess deaths, mostly among the elderly (Bridges et al. 2003). Influenza 
vaccination has been shown to significantly reduce influenza-related morbidity and mortality (Bridges et al. 2003), and the Advisory Committee on Immunization Practices recommends that nearly all persons over the age of 50 receive annual vaccination (Bridges et al. 2003). Since 1993, Medicare has covered the full cost of influenza vaccination.

While the Healthy People 2000 goal of 60 percent influenza vaccination among the elderly has been met at the national level, significant racial disparities persist (CDC 2003a, b). Behavioral Risk Factor Surveillance System (BRFSS) data for 2000 and 2001 combined indicate rates of 66.0 percent among elderly non-Hispanic whites, $48.3 \%$ among non-Hispanic blacks, and 53.7 percent among Hispanics (CDC 2003a). National Health Interview Survey data indicate similar rates in 2002 of $69.6,48.5$, and 50.7 percent, respectively (CDC 2003b). Elimination of these disparities is a goal of Healthy People 2010 (HHS 1999) and the Racial and Ethnic Adult Disparities in Immunization Initiative (READII) at the Department of Health and Human Services (HHS 2002). This work is made more difficult because the causes of the disparity are poorly understood and the effectiveness of strategies to increase immunizations has not been established in minority populations. Metaanalyses (Szilagyi et al. 2000; Stone et al. 2002) have documented the effectiveness of both patient-focused strategies, such as mailed reminders and patient education, and clinic-based strategies, such as providing physician reminders and incentives, allocating space and personnel at clinics for the purpose of providing vaccinations, and empowering nurses to provide influenza vaccinations. However, many of these studies were conducted in areas with low minority populations, or used administrative databases that do not identify the race of the beneficiary. How well these strategies will work in minority populations, which may have different barriers to overcome, is not clear. For example, organizational changes at a clinic will have little effect on populations that access clinical care infrequently, and postcard reminders may have little effect on populations with resistant attitudes or beliefs regarding vaccination.

Address correspondence to Paul L. Hebert, Ph. D., Assistant Professor, Mount Sinai School of Medicine, Department of Health Policy, New York, NY. Kevin D. Frick, Ph. D., Associate Professor, is with the Johns Hopkins Bloomberg School of Public Health, Department of Health Policy and Management, Baltimore, MD. Robert L. Kane, Ph. D., Professor and Minnesota Chair in Long Term Care and Aging, is with the University of Minnesota School of Public Health, Division of Health Services Research and Policy, Minneapolis, MN. A. Marshall McBean, M.D., M.Sc., Professor, is with the University of Minnesota School of Public Health, Division of Health Services Research and Policy, Minneapolis. 
The purpose of this study was to identify the causes of racial and ethnic disparities in influenza vaccination in the elderly in order to better inform efforts to eliminate the disparity. We explored three possible causes of the disparity: (1) differences in resistant attitudes and beliefs regarding influenza vaccination, (2) differences in access to care during weeks when influenza vaccinations were given, and (3) discriminatory treatment of African-American and Hispanic patients by their providers.

\section{METHODS}

Data were from the Medicare Current Beneficiary Survey (MCBS) (Adler 1994) Access to Care and accompanying Medicare claims files for 1995 through 1996. The MCBS is an annual, in-person, rotating panel survey that elicits information on the health, health behaviors, and use of medical services for a panel of several thousand Medicare beneficiaries nationwide. We studied community-dwelling respondents $\geq 65$ years who completed both the 1995 and 1996 surveys. We defined vaccination status for the 1995-1996 influenza season by self-reported influenza vaccination use, which was elicited in the 1996 survey. Self-reported vaccine use is highly sensitive but only modestly specific when compared with medical records (Nichol, Korn, and Baum 1991; Mac Donald et al. 1999; Zimmerman, Raymund et al. 2003). We defined three racial groups according to the beneficiary's self-reported race and ethnicity: non-Hispanic white, non-Hispanic African American, and Hispanic. For the remainder of the paper, the first two will be referred to as "white" and as "African American," respectively.

\section{Resistant Attitudes and Beliefs}

To address whether resistant attitudes and beliefs regarding influenza vaccination are a cause of racial disparities, we analyzed self-reported reasons for not receiving a vaccination. In the $1996 \mathrm{MCBS}$, participants who self-reported that they did not receive an influenza vaccination in 1995 were asked for all reasons they had not been vaccinated (CDC 1999). Interviewers assigned each reason to one of 13 categories (Table 1). We subjectively classified each category as reflecting resistance to vaccination if it suggested attitudes or beliefs about influenza vaccinations that might inhibit a beneficiary from accepting a free vaccination if one were offered. In a recent study, persons who agreed with statements in any of these resistant categories were far less likely to receive vaccination than were persons who did not hold these beliefs 


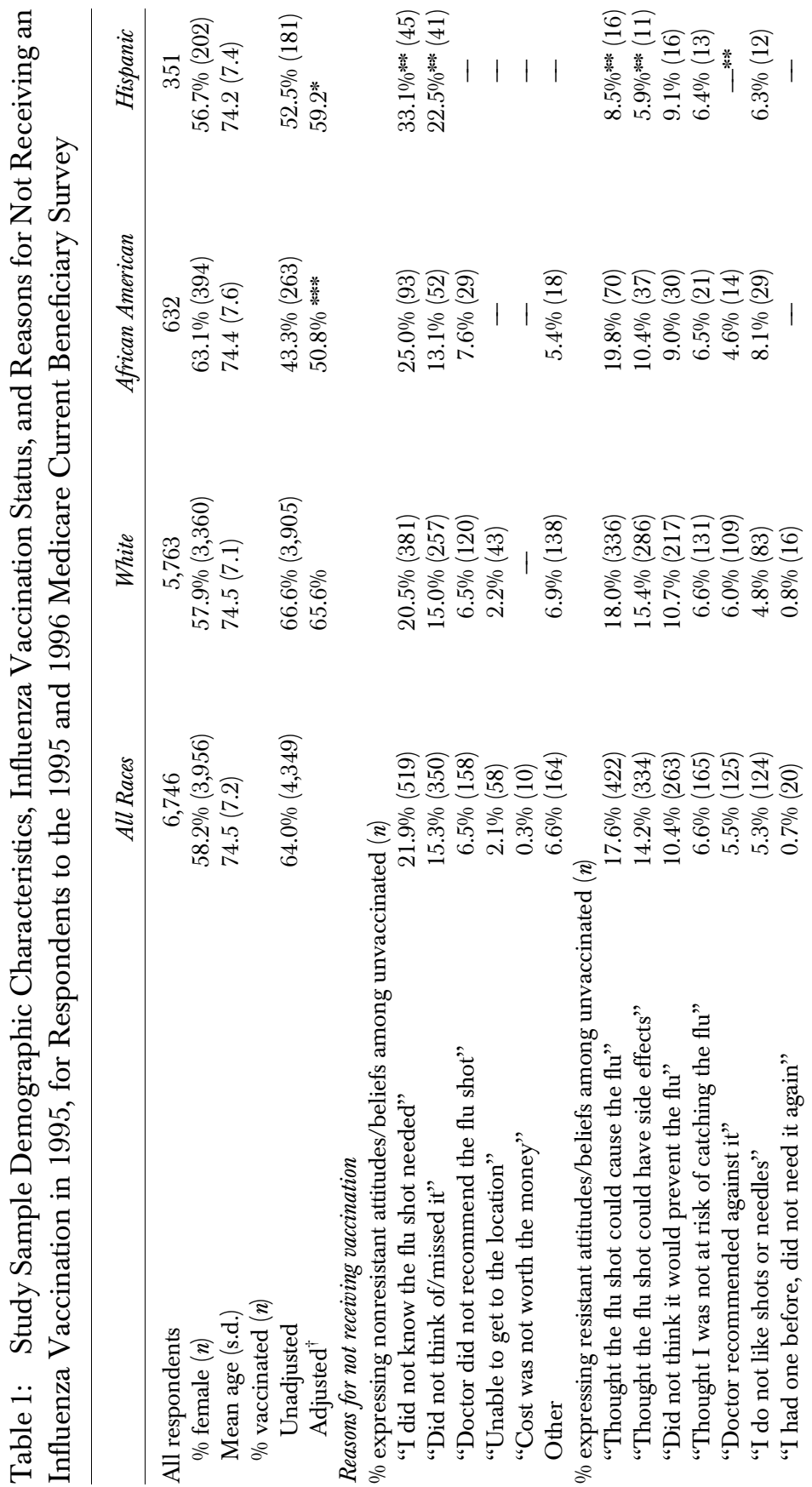


$\Re$

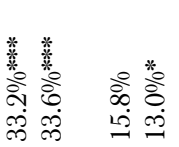

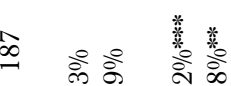
मीं

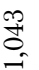

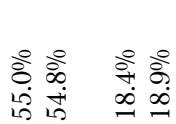

ป

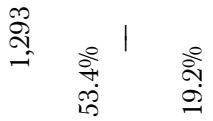
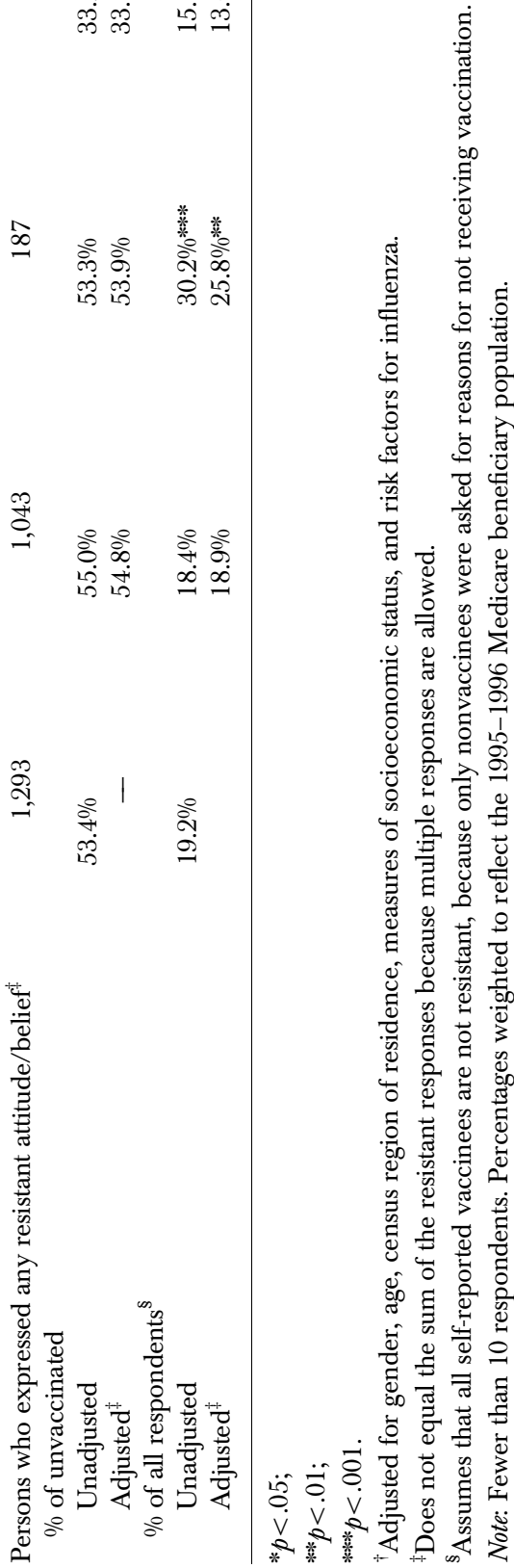
(Santibanez et al. 2002), although the study did not specifically address the role of race. We used this classification to code every MCBS participant as resistant or not resistant to vaccination. Persons who gave any resistant response were coded resistant. All others were coded as nonresistant. This had the effect of coding all self-reported vaccinees as nonresistant because only those who reported that they had not received vaccination were asked reasons for remaining unvaccinated.

\section{Access to Care}

To explore the role of access to care, we used Medicare claims to identify opportunities for vaccination of MCBS respondents. Diagnosis codes for influenza vaccination (ICD-9-CM code V048) on Physician/Supplier Part B claims and hospital outpatient claims revealed that 90 percent of vaccinations were provided in the 38 th-49th weeks of 1995 . We coded each beneficiary as having the opportunity to receive a vaccination if during these weeks he/she had a hospital outpatient claim, or had a Physician/Supplier claim for either a medical service, or a nonmedical service that was provided in a location where vaccinations are given.

\section{Provider Discrimination}

Discrimination is defined by differences in the offering of vaccination according to the race/ethnicity of the patient. Unfortunately, we observe the receipt of vaccination, not the offering, and a beneficiary may not receive vaccination for reasons other than discrimination. We attempt to control for these reasons by creating a cohort of white and nonwhite beneficiaries who received care from the same set of providers during weeks when influenza vaccinations were given, and who had not received vaccination from a different provider. To create this cohort, we identified the "usual" provider for each African-American and Hispanic beneficiary as the provider tax code on Physician/Supplier Part B claims responsibility for the plurality of claims for medical (nonlaboratory) services in 1995. We identified African-American and Hispanic beneficiaries who had a medical encounter with their respective usual provider during influenza vaccination weeks and matched them to white beneficiaries who had a medical encounter with the same providers during those weeks. Because beneficiaries might have refused a vaccination on one of these visits because they had already received vaccination from another provider, we identified self-reported vaccinees who did not generate a Medicare claim for vaccination from their respective "usual" providers, and 
eliminated them from the cohort. To increase the sample size, we constructed similar cohorts for the vaccination weeks in years 1993 and 1994. We then identified vaccinations that were delivered at one of these encounters using diagnosis codes on Part B Physician/Supplier claims.

Racial differences in vaccinations on these encounters could occur if white beneficiaries were more likely to initiate the encounter for the purpose of vaccination. To account for this, we distinguished two types of vaccinations: those that were delivered as the primary reason for the medical encounter and those that were delivered as an adjunct to some other medical encounter. The first diagnosis field on the Physician/Supplier claim identifies the diagnosis that precipitated the encounter. If the diagnosis code for influenza vaccination appeared in the first position on the Physician/Supplier claim, we said that the patient had a "vaccination-initiated" encounter. If the code for vaccination appeared in a secondary position, we said the beneficiary received an "adjunct vaccination." Finally, because differences in the complexity of the medical encounter may affect the likelihood that an adjunct vaccination is offered, we report the mean reimbursement per Physician/Supplier claim, and the mean number of line items (i.e., separate procedures) per claim, for claims in vaccination weeks, by race.

We used longitudinal sampling weights to calculate all percentages, and estimated means, standard errors, and design-based $\chi^{2}$ tests in a manner consistent with the complex sampling structure of the MCBS (O'Connell, Chu, and Bailey 1997) using Stata 7.0 (Stata 1999). All p-values are based on comparisons with the white rate. Race-specific adjusted rates for binary outcomes were calculated by estimating logistic regressions. Covariates included race/ ethnicity (African American, Hispanic), gender, age, census region of residence, education, income, marital status; indicators for rural residence, Medicaid "buy-in" status, and status as a Medigap policy holder; and measures of health status (indicators for self-reported cancer, diabetes, heart disease, hypertension, and respiratory disease; indicators of general health in five categories from poor to excellent; the number of activities of daily living and instrumental activities of daily living that were limited by health; and smoking status). We derived adjusted rates for African Americans by setting the indicator variable for African-American race equal to one and the Hispanic indicator variable to zero, and then calculating the predicted probability for each individual using the coefficients from the logistic regression. The survey-weighted mean of these predicted probabilities gave the adjusted rate for African Americans. We used a Taylor series approximation to calculate the standard error of prediction (Green 1993). We used similar 
techniques to calculate the white and Hispanic adjusted rates and confidence intervals.

\section{RESULTS}

In 1995 and 1996, 8,562 elderly Medicare beneficiaries completed all rounds of MCBS interviews. We eliminated 748 who were in a nursing home or other facility, and 54 who could not recall whether they received an influenza vaccination for the 1995-1996 influenza season. Because Medicare claims are not generally reported for persons in managed care plans, we eliminated 882 with any managed care participation in 1995 . We also dropped 132 beneficiaries who were not white, African American, or Hispanic, because of small cell sizes for this group in many of the analyses. The final sample included 6,746 persons; 632 (9.4 percent) were African American and 351 (5.2 percent) were Hispanic (Table 1). Influenza vaccination rates were significantly lower for African-American (43.3 percent; $p<0.001)$ and Hispanic (52.5 percent; $p=0.003)$ beneficiaries than for white beneficiaries ( 66.6 percent).

\section{Resistant Attitudes and Beliefs}

Table 1 groups reasons for not receiving an influenza vaccination by whether or not they suggest a resistance to vaccination. The most common nonresistant reason given was lack of knowledge that influenza vaccinations were recommended (21.9 percent), a response given significantly more often by Hispanic beneficiaries (33.1 percent, $p=0.006$ ). The most common resistant reasons were the misperception that vaccination could cause influenza (white 18.0 percent; African American 19.8 percent, $p>0.20$; Hispanic 8.5 percent, $p=0.002$ ), and an aversion to perceived side effects (white 15.4 percent; African American 10.4 percent, $p>0.20$; Hispanic 5.9 percent, $p=0.007$ ). Among the unvaccinated, resistance was similar for white (55.0 percent) and African-American beneficiaries (53.3 percent, $p=0.615$ ), but significantly lower for Hispanic beneficiaries (33.2 percent, $p<0.001$ ). If persons who selfreported that they received vaccination are considered not resistant to vaccination, then overall resistance was significantly higher among AfricanAmerican beneficiaries (30.2 percent, $p<0.001)$ than white beneficiaries $(18.4$ percent). Thus, resistance may explain as much as 11.8 percentage points $(30.2-18.4 \%=11.8 \%)$ of the difference in white and African-American vaccination rates. However, there was no evidence that resistance among 
Hispanic beneficiaries (15.8 percent, $p=0.357$ ) contributed to low vaccination rates compared with whites.

\section{Access to Care}

Racial disparities in access to care existed in the weeks before influenza vaccination season (Table 2, row 2) and continued during vaccination weeks (row 3). The disparity during vaccination weeks was only partly because of higher vaccination-initiated encounters by white beneficiaries (Table 2, row 5): excluding vaccination-initiated encounters (that is, encounters for which vaccination was the first listed code on the Physician/Supplier claim), the percentage of beneficiaries who had a medical encounter during influenza vaccination weeks was higher for white (68.5 percent), than African-American (60.8 percent, $p<0.001$ ), or Hispanic (60.1 percent, $p=0.003$ ) beneficiaries, although differences in the adjusted rates were not statistically significantly. There was no significant racial difference in access to health care among unvaccinated beneficiaries: roughly half of all unvaccinated beneficiaries had medical encounters during influenza vaccinations weeks, regardless of race.

\section{Provider Discrimination}

We identified 278 African-American and 83 Hispanic beneficiaries who had a medical encounter with their "usual" provider during weeks when influenza vaccinations were given, and matched them with 1,127 white beneficiaries who had a medical encounter with one of these same providers during the same weeks (Table 3, row 1). We eliminated 514 beneficiaries who received an influenza vaccination from another provider, resulting in a final sample of 974 beneficiaries.

Even among beneficiaries visiting the same provider during the same weeks, white beneficiaries were still substantially more likely to receive a Medicare-paid vaccination than were African-American beneficiaries (white 70.4 percent; African American 35.1 percent, $p<0.001$; Hispanic 61.1 percent, $p=0.168$ ). However, these differences were largely explained by much higher vaccination-initiated visits by white beneficiaries. White beneficiaries were $>5$ times as likely as African Americans (42.0 percent white; 8.2 percent African American, $p<0.001)$ and 1.6 times as likely as Hispanic beneficiaries (25.9 percent, $p=0.009)$ to receive a Medicare-paid vaccination where vaccination was the first-listed code on the claim. On 92 percent of vaccination-initiated encounters, influenza vaccination alone, or influenza 


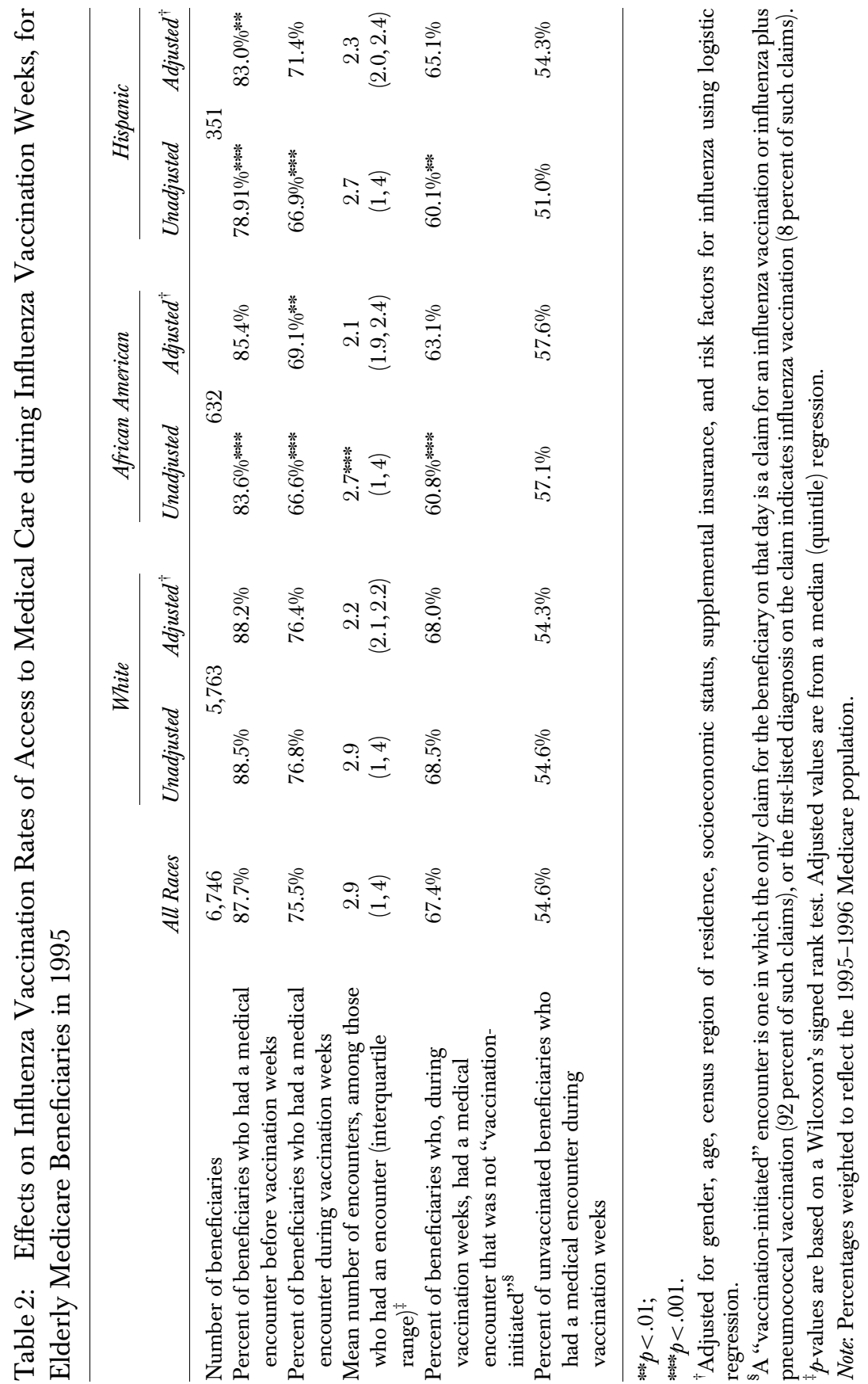




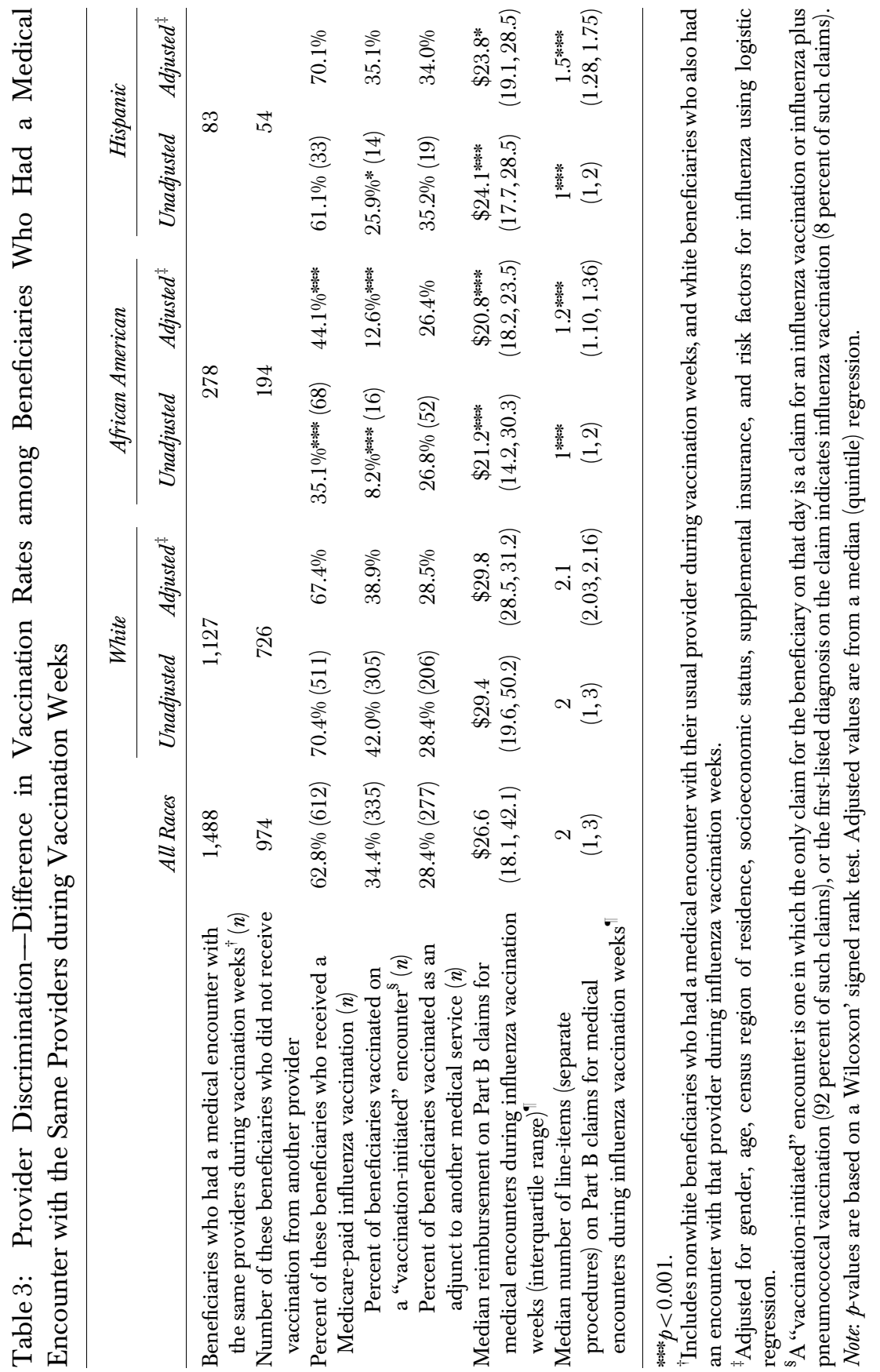


plus pneumococcal vaccination, were the only procedures given that beneficiary on that day.

The adjunct vaccination rate did not differ substantially by race (Table 3 , row 5): among beneficiaries who did not initiate the encounter for the purpose of vaccination, 28.4 percent received a vaccination as an adjunct to another medical service, and this rate did not differ substantially by race. The average reimbursement per claim, and the number of individual procedures given to beneficiaries during vaccination weeks were higher for white beneficiaries (Table 3, rows 6-7). Although a simple count of the number and value of procedures on an encounter is a crude measure of complexity, we found little evidence to suggest that physicians failed to remind their minority patients about vaccination because of especially busy visits with these patients.

\section{Summary of Causes of Racial Differences in Influenza Vaccination}

Table 4 summarizes the causes of racial disparities in influenza vaccination that we discussed above. For this analysis we returned to the entire sample of 6,746 respondents to the 1995-1996 MCBS, rather than the subset of patients who were seen in the same providers' offices. The first section of Table 4 shows the number and percent of beneficiaries who were not vaccinated in 1995. These beneficiaries are further classified according to whether they expressed resistance to vaccination and/or they did not make an encounter with a medical provider during vaccination weeks. The second row shows that a significant percentage of African-American (13.7 percent; $p<0.001)$ and Hispanic $(16.8$ percent; $p<0.001)$ beneficiaries remain unvaccinated despite being in providers' offices during vaccination weeks and having no stated resistance to vaccination. These represent missed opportunities for vaccination that could be addressed through greater vigilance on the part of providers. Missed opportunities are substantially higher for minority beneficiaries than for white beneficiaries (7.6 percent).

The remaining rows in the first section of Table 4 represent beneficiaries for whom more effort would have been required to change their vaccination status. The second row shows people who were in a provider's office, but expressed resistance to vaccination. These are beneficiaries who needed to be disabused of their resistance, but did not need motivation to make an appointment with their provider, since they were already in a provider's office during vaccination weeks. Because resistance in general is higher among African-American beneficiaries, this category is large for African-American 


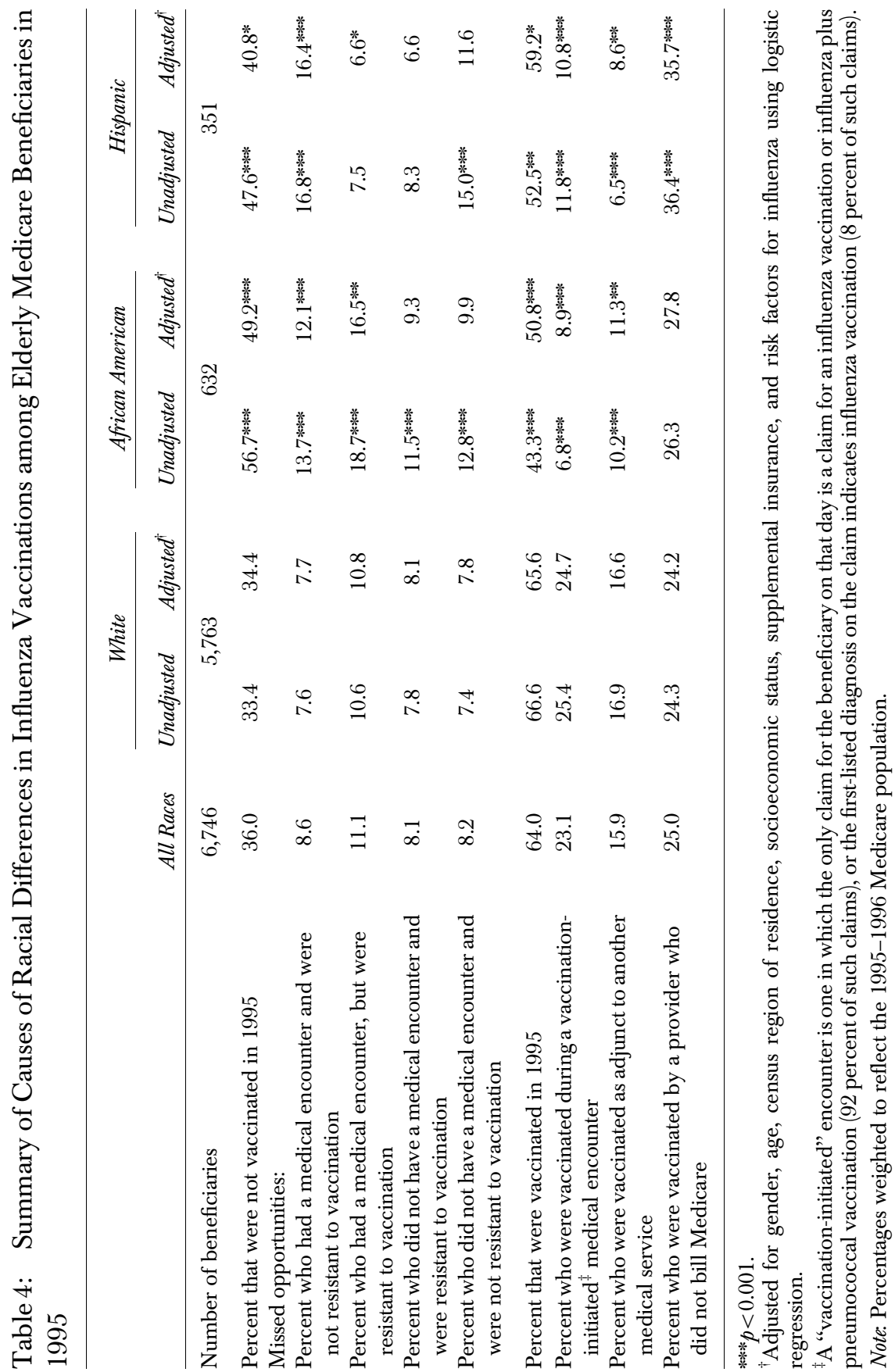


beneficiaries 18.7 percent $(p<0.001)$, but less so for Hispanic (7.5 percent; $p=0.06)$ or white (10.6 percent) beneficiaries. The remaining categories in the first section of Table 4 are people who did not have a medical encounter during vaccination weeks. Care should be taken in interpreting the higher percentages of nonwhite beneficiaries in these categories as evidence that access plays a major role in racial disparities in vaccination. The higher percentages are partly a reflection of the higher percentage of nonvaccinees in minority groups-even if access were entirely unrelated to vaccination, one would expect to see more unvaccinated minorities who did not have a medical encounter, simply because there are more unvaccinated minority beneficiaries. In fact, as was shown in Table 2 , access to care that was not vaccine-initiated was fairly similar across race groups. The higher percentages are also partly a consequence of vaccine-initiated medical encounters by white beneficiaries. That is, higher access for white beneficiaries is partly a consequence of, not a cause of, higher white vaccination rates.

The second section of Table 4 shows the percent of beneficiaries who did receive vaccination in 1995, and how they obtained their vaccination. As was found in the analysis of provider discrimination discussed above (Table 3), white beneficiaries in this sample were significantly more likely to receive vaccination on a vaccine-initiated encounter (25.4 percent) than were African-American (6.8 percent; $p<0.001)$ or Hispanic (11.8 percent; $p<0.001)$ beneficiaries. Among white vaccinees, 38 percent $(25.4 / 66.6=38.1)$ received vaccination on a vaccine-initiated encounter, compared with 16 and 22 percent of African-American and Hispanic vaccinees, respectively. However, in contrast to the earlier analysis of provider discrimination (Table 3), the rate of vaccination given as an adjunct to some other medical service was also higher in whites (16.9 percent) than AfricanAmerican (10.2 percent; $p<0.001)$ or Hispanic (6.5 percent; $p<0.001)$ beneficiaries. That is, although we found large racial differences in adjunct vaccinations in the general population of beneficiaries, we found none in the analysis of provider discrimination in which we constrained the sample to include only beneficiaries who shared the same usual provider. This may reflect a situation in which minority beneficiaries tend to frequent providers who give fewer adjunct vaccinations, but that no individual provider discriminates.

Table 4 also shows that Hispanic beneficiaries are more likely to selfreport receiving an influenza vaccination, but to generate no Medicare claim for vaccination. 


\section{DISCUSSION}

This study increases our understanding of racial disparities by analyzing data on medical encounters during vaccination weeks and stated reasons for not receiving vaccination. Previous studies of racial differences in influenza vaccination rates have attempted to explain the difference in vaccination rates by adjusting for other factors known to be correlated with vaccination use (Gornick et al. 1996; Fowles and Beebe 1998; Schneider et al. 2001; Egede and Zheng 2003). In general, these studies found that racial disparities persisted after controlling for a long list of other factors, including measures of socioeconomic status, risk factors for influenza, access to health care, characteristics of the health insurance coverage, and attitudes toward health care.

\section{Attitudes and Beliefs toward Influenza Vaccinations}

Resistant attitudes and beliefs were more prevalent among African Americans than either white or Hispanic beneficiaries, and this accounted for part of the disparity among African Americans. However, these estimates derive from the assumptions that all vaccinees were not resistant, and that all persons who had a resistant attitude would refuse vaccination if it were offered. These assumptions may overemphasize the role of attitudes in influencing the decision to seek vaccination. Since only unvaccinated beneficiaries were asked why they did not receive vaccination, we could not observe whether vaccinees also had some aversions to vaccination.

Other researchers have addressed this question with mixed results. One recent study found a very strong correlation between resistant attitudes and vaccination behavior, with 72 percent of the unvaccinated expressing some concern about effectiveness and side effects of influenza, compared with only 11 percent of vaccinees (Zimmerman, Santibanez et al. 2003). Another (van Essen, Kuyvenhoven, and de Melker 1997) found similar strong influences of beliefs of side effects and personal risk of influenza. However, a study among predominantly white men (Nichol, MacDonald, and Hauge 1996) found a 70.4 percent influenza vaccination rate among respondents who believed that vaccination could cause influenza, and 32.7 percent vaccination rate among persons who thought they personally would get sick from flu shots. Moreover, if resistance were a major cause of low African-American vaccination rates, one would expect a relatively high percentage of African Americans to refuse vaccinations offered as an adjunct to another service, resulting in a lower adjunct vaccination rate relative to whites, and we did not find this in the 
analysis of provider discrimination. Thus, the contribution of resistance to low African-American vaccination rates may be less than we estimate. We found no evidence that resistance contributes to the lower vaccination rates of Hispanic beneficiaries. In fact, Hispanic beneficiaries expressed less resistance than white beneficiaries.

\section{Access to Care}

Access was not a significant cause of racial disparities. Excluding vaccineinitiated encounters, access to care during vaccination weeks was about 8 percentage points higher for whites. However, access alone does not guarantee vaccination. We found that 67.4 percent of all beneficiaries had a medical encounter that was not vaccine-initiated (Table 2), and 15.9 percent of beneficiaries received a vaccination as an adjunct to an unrelated medical encounter (Table 4). Thus, only 23.6 percent (15.9/67.4) of people with an encounter received vaccination as an adjunct to medical services received on one of those encounters. If routine access to care were equivalent between race groups, we would expect African-American and Hispanic vaccination rates to increase by $<2$ percentage point (e.g., $8 \times 23.6 \%=1.9 \%$ ). These findings are consistent with a previous study (Santibanez et al. 2002) that showed that access to care was not related to vaccination status.

However, a pronounced disparity existed among beneficiaries who had an encounter during vaccination weeks and had no resistance to vaccination. African-American and Hispanic beneficiaries were almost twice as likely as white beneficiaries to remain unvaccinated despite being nonresistant and in a provider's office during vaccination weeks (Table 4). This is a clear failure on the part of providers to give proven, effective preventive care to minority patients. Eliminating these missed opportunities for vaccination could substantially improve vaccination rates for African Americans (+13.7 percent), Hispanics ( +16.8 percent), and whites ( +7.6 percent). This would raise vaccination rates in each race group to rates that are statistically no different than the Public Health 2000 goal of 60 percent vaccination. That is, by simply offering vaccination to nonresistant beneficiaries who are already in a provider's office, the Public Health 2000 goal could have been achieved 5 years ahead of schedule, in all three race groups. This argues strongly for education programs for providers who serve minority beneficiaries, and for studies into the barriers to implementing vaccination programs among these providers. 


\section{Reasons Beneficiaries Remain Unvaccinated}

Why do such large percentages of African-American and Hispanic beneficiaries remain unvaccinated despite having no resistance and despite having an encounter with a provider during vaccination weeks? One possibility is racial discrimination by providers. Indeed, we discovered that African-American and Hispanic beneficiaries were not being vaccinated despite having medical encounters with their usual providers on days when those same providers were administering vaccinations to white beneficiaries. While this seemed at first to be strong evidence of prejudicial treatment, a closer analysis revealed that the disparity in vaccine use was essentially attributable to a much higher rate of vaccine-initiated encounters among white patients. White beneficiaries were $>5$ times as likely as African-American and 1.6 times as likely as Hispanic beneficiaries to make a trip to their provider for the primary and often sole purpose of obtaining a vaccination. This might still reflect discrimination if providers are reminding only their white patients to return for vaccination, but if this were true, we would also expect to see racial differences in adjunct vaccinations, and we did not. However, these null findings regarding adjunct vaccinations should be interpreted carefully; with only 194 African-American beneficiaries available for this analysis, we had only 25 percent power to detect a 5 percentage point difference in adjunct vaccination rates. Further analysis of this issue, with a database of sufficient size, is clearly required.

Future studies should also address the extent to which racial differences in adjunct vaccinations are attributable minorities frequenting providers who have lower propensities to offer adjunct vaccinations. This could be explored by assessing racial disparities in vaccinations across providers stratified by the percent of minorities treated by the provider. If providers who treat a large percentage of minorities offer proportionally fewer vaccinations to minorities and nonminorities alike, then a key cause of the low rate of adjunct vaccinations among African-American and Hispanic beneficiaries is not discrimination, but inequitable distribution of these beneficiaries to providers with effective vaccination programs. We were unable to address this issue because the MCBS contains only a small fraction of the beneficiaries treated by any given provider.

This study highlights the importance of the motivated patient. Vaccines delivered on vaccine-initiated visits imply that the patient was willing to make a special trip to receive vaccination. Why white beneficiaries showed more self-motivation, or why the message to receive a vaccination resonated more with white than nonwhite beneficiaries is not clear. Vaccinees tended to act on 
the provider reminder or public health message, or they felt empowered to seek an influenza vaccination without having to be reminded. Perhaps, public health marketing efforts need to be more socially and culturally targeted to encourage more African-American and Hispanic beneficiaries to seek vaccinations. Other studies conducted in largely white populations (Becker and Maiman 1975; Becker et al. 1977; Buchner, Carter, and Inui 1985; Carter et al. 1986; Nichol, MacDonald, and Hauge 1996) have shown that this intention to receive vaccination is related to a number of factors, including the knowledge that vaccination is recommended, the perceived susceptibility to and severity of influenza, and the belief that vaccination prevents influenza. Future studies should assess which of these motivational factors contribute to the low rate of vaccine-initiated encounters in minority populations.

This study also highlights the need to tailor interventions to the specific needs of the community. Because resistant attitudes and beliefs played a role in relatively low vaccination rates for African Americans, solutions that rely heavily on patient or provider reminders may be less effective in AfricanAmerican communities. Further research is necessary on the origins of these resistant attitudes and the extent to which they can be disabused by better provider-patient communication. Can resistance be overcome if providers offer African-American beneficiaries a vaccination while assuring them that it is safe and effective, or are the roots of the resistance in a general lack of trust that would require long-term improvements in provider-patient communication among African-Americans patients? In contrast to African Americans, Hispanics expressed little resistance, but Hispanic vaccinees were considerably less likely to have generated a Medicare claim for vaccination. Although this may represent over-reporting of vaccination among Hispanic beneficiaries, it may demonstrate a preference among Hispanics to receive vaccination from providers who typically do not bill Medicare, such as providers at community health fairs or other influenza outreach programs. Perhaps the best way to reach Hispanic beneficiaries is not through their medical providers.

There are several limitations. First, the data are several years old, and, although the disparity in vaccination still exists, the underlying causes may have changed. Second, we rely on self-reported vaccination, which, because it has high sensitivity but modest specificity, would tend to overestimate vaccination rates. Whether the overestimation differs by race for influenza vaccination is unknown, although studies have found that self-reported measures inflate true rates of pap smears and mammograms among racial minorities (Zapka et al. 1996; McPhee et al. 2002). Finally, the sample size for the 
assessment of provider discrimination is too small to rule out discrimination in the delivery of vaccinations as an adjunct to another medical service.

\section{CONCLUSION}

Resistant attitudes and beliefs contribute to relatively low vaccination rates for African-American, but not Hispanic beneficiaries. Access to care plays a small role in racial and ethnic differences in vaccination rates. Although our sample size was limited, we found no evidence of provider discrimination in the administration of influenza vaccination. The first and lowest barrier to improved influenza vaccination use is the elimination of missed opportunities for vaccination among beneficiaries who already make contact with the health care system and have no stated aversion to immunization. We estimate that had every minority beneficiary who made contact with the health care system in the fall of 1995 been offered an influenza vaccination, the proposed Healthy People 2000 goal of 60 percent vaccination would have been achieved in three major race/ethnic groups 5 years ahead of schedule.

\section{ACKNOWLEDGMENTS}

Support for this research was provided by the Agency for Healthcare Research and Quality (Grant number R03-HS10154-01). Helpful comments were provided by Jane E. Sisk, Ph.D., Leah Tuzzio, M.P.H., and two anonymous reviewers.

\section{REFERENCES}

Adler, G. 1994. "A Profile of the Medicare Current Beneficiary Survey.” Health Care Financing Review 15: 153-63.

Becker, M. H., D. P. Haefner, S. V. Kasl, J. P. Kirscht, L. A. Maiman, and I. M. Rosenstock. 1977. "Selected Psychosocial Models and Correlates of Individual Health-Related Behaviors." Medical Care 15 (5, suppl): 27-46.

Becker, M., and L. Maiman. 1975. "Sociobehavioral Determinants of Compliance with Health and Medical Care Recommendations." Medical Care 13 (1): 10-24.

Bridges, C. B., S. A. Harper, K. Fukuda, T. M. Uyeki, N. J. Cox, and J. A. Singleton. 2003. "Prevention and Control of Influenza. Recommendations of the Advisory Committee on Immunization Practices (ACIP)." Morbidity and Mortality Weekly Report 52 (Recomm Rep-8): 1-38. 
Buchner, D. M., W. B. Carter, and T. S. Inui. 1985. "The Relationship of Attitude Changes to Compliance with Influenza Immunization. A Prospective Study." Medical Care 23 (6): 771-9.

Carter, W. B., L. R. Beach, T. S. Inui, J. P. Kirscht, and J. C. Prodzinski. 1986. "Developing and Testing a Decision Model for Predicting Influenza Vaccination Compliance." Health Services Research 20 (6, part 2): 897-932.

CDC. 1999. "Reasons Reported by Medicare Beneficiaries for Not Receiving Influenza and Pneumococcal Vaccinations-United States, 1996." Morbidity and Mortality Weekly Report 48 (39): 556-890.

CDC. 2003a. "Racial/Ethnic Disparities in Influenza and Pneumococcal Vaccination Levels among Persons Aged > or = 65 Years-United States, 1989-2001." Morbidity and Mortality Weekly Report 52 (40): 958-62.

CDC. 2003b. "Early Release of Selected Estimates Based on Data from January-June 2003," National Health Interview Survey. Available at http://www.cdc.gov/ nchs/data/nhis/earlyrelease/200312_04.pdf [accessed March 14, 2004]

Egede, L. E., and D. Zheng. 2003. "Racial/Ethnic Differences in Adult Vaccination among Individuals with Diabetes." American Journal of Public Health 93 (2): 324-9.

Fowles, J. B., and T. J. Beebe. 1998. "Failure to Immunize the Elderly: A Systems Problem or a Statement of Personal Values?" Joint Commission Journal on Quality Improvement 24 (12): 704-10.

Gornick, M. E., P. W. Eggers, T. W. Reilly, R. M. Mentnech, L. K. Fitterman, L. E. Kucken, and B. C. Vladeck. 1996. "Effects of Race and Income on Mortality and Use of Services among Medicare Beneficiaries." New England Journal of Medicine 335 (11): 791-9.

Green, W. 1993. Econometric Analysis, 645 pp. Englewood Cliffs, NJ: Prentice-Hall, Inc. HHS. 1999. The Initiative to Eliminate Racial and Ethnic Disparities in Health. Washington, DC: U.S. Department of Health and Human Services.

HHS. 2002. "Initiative to Reduce Racial and Ethnic Disparities in Adult Immunizations" Available at http://raceandhealth.hhs.gov [accessed January 21, 2005].

Mac Donald, R., L. Baken, A. Nelson, and K. L. Nichol. 1999. "Validation of SelfReport of Influenza and Pneumococcal Vaccination Status in Elderly Outpatients." American Journal of Preventive Medicine 16 (3): 173-7.

McBean, A. M., J. D. Babish, and J. L. Warren. 1993. "The Impact and Cost of Influenza in the Elderly." Archives of Internal Medicine 153 (18): 2105-11.

McPhee, S. J., T. T. Nguyen, S. J. Shema, B. Nguyen, C. Somkin, P. Vo, and R. Pasick. 2002. "Validation of Recall of Breast and Cervical Cancer Screening by Women in an Ethnically Diverse Population." Preventive Medicine 35 (5): 463-73.

Nichol, K. L., J. E. Korn, and P. Baum. 1991. "Estimation of Outpatient Risk Characteristics and Influenza Vaccination Status: Validation of a Self-Administered Questionnaire.” American Journal of Preventive Medicine 7 (4): 199-203.

Nichol, K. L., R. Mac Donald, and M. Hauge. 1996. "Factors Associated with Influenza and Pneumococcal Vaccination Behavior among High-Risk Adults.” Journal of General Internal Medicine 11 (11): 673-7. 
O’Connell, J. O., A. Chu, and R. C. Bailey. 1997. "Considerations for Analysis of Medicare Current Beneficiary Survey (MCBS) across Time." Proceedings of the Section on Survey Research Methods; American Statistical Review. Available at http://www.amstat.org/sections/srms/proceedings/papers/1997_110.pdf.

Santibanez, T. A., M. P. Nowalk, R. K. Zimmerman, I. K. Jewell, I. J. Bardella, S. A. Wilson, and M. A. Terry. 2002. "Knowledge and Beliefs about Influenza, Pneumococcal Disease, and Immunizations among Older People.” Journal of American Geriatric Society 50 (10): 1711-6.

Schneider, E. C., P. D. Cleary, A. M. Zaslavsky, and A. M. Epstein. 2001. "Racial Disparity in Influenza Vaccination: Does Managed Care Narrow the Gap between African Americans and Whites?" Journal of the American Medical Association 286 (12): 1455-60.

Stata. 1999. Stata 6.0. College Station, TX: Stata Corporation.

Stone, E. G., S. C. Morton, M. E. Hulscher, M. A. Maglione, E. A. Roth, J. M. Grimshaw, B. S. Mittman, L. V. Rubenstein, L. Z. Rubenstein, and P. G. Shekelle. 2002. "Interventions That Increase Use of Adult Immunization and Cancer Screening Services: A Meta-Analysis." Annals of Internal Medicine 136 (9): 641-51.

Szilagyi, P. G., C. Bordley, J. C. Vann, A. Chelminski, R. M. Kraus, P. A. Margolis, and L. E. Rodewald. 2000. "Effect of Patient Reminder/Recall Interventions on Immunization Rates: A Review." Journal of the American Medical Association 284 (14): 1820-7.

van Essen, G. A., M. M. Kuyvenhoven, and R. A. de Melker. 1997. “Compliance with Influenza Vaccination. Its Relation with Epidemiologic and Sociopsychological Factors." Archives of Family Medicine 6 (2): 157-62, discussion 163.

Zapka, J. G., C. Bigelow, T. Hurley, L. D. Ford, J. Egelhofer, W. M. Cloud, and E. Sachsse. 1996. "Mammography Use among Sociodemographically Diverse Women: The Accuracy of Self-Report." American Journal of Public Health 86 (7): $1016-21$.

Zimmerman, R. K., M. Raymund, J. E. Janosky, M. P. Nowalk, and M. J. Fine. 2003. "Sensitivity and Specificity of Patient Self-Report of Influenza and Pneumococcal Polysaccharide Vaccinations among Elderly Outpatients in Diverse Patient Care Strata." Vaccine 21 (13-14): 1486-91.

Zimmerman, R. K., T. A. Santibanez, J. E. Janosky, M. J. Fine, M. Raymund, S. A. Wilson, I. J. Bardella, A. R. Medsger, and M. P. Nowalk. 2003. "What Affects Influenza Vaccination Rates among Older Patients?" American Journal of Medicine 114 (1): 31-8. 
